\title{
ARTÍCULOS
}

\section{NOTAS HISTÓRICAS Y EPISTEMOLÓGICAS SOBRE EL CAMPO DE LA POLÍTICA EDUCATIVA EN LATINOAMÉRICA}

Cesar Tello ${ }^{1}$ (D)

RESUMEN: En este trabajo se presentan algunas notas históricas y epistemológicas del campo de la política educativa en Latinoamérica. Haciendo especial hincapié en el enfoque de las epistemologías de la política educativa como un modo de análisis de las investigaciones que los investigadores llevan a cabo en el campo. Este artículo tiene la intención de re pensar el campo de las políticas educativas en el marco de las transformaciones y crisis de las ciencias sociales y políticas ante la necesidad de comprender y nombrar la compleja realidad socio educativa actual.

Palabras clave: Política educativa. Epistemología. Investigación educativa. Investigadores. América Latina.

\section{HISTORICAL AND EPISTEMOLOGICAL NOTES ON THE FIELD OF EDUCATIONAL POLICY IN LATIN AMERICA}

\begin{abstract}
In this paper, we present some historical and epistemological notes about the education policy field in Latin America, emphasizing the focus of education policy epistemologies as a mode of analysis of the researches conducted by field professionals. This article intends to rethink the field of education policy under the transformations and crises of social and political sciences at the necessity to understand and name the current complex socio educational reality.
\end{abstract}

Keywords: Educational policy. Epistemology. Educational research. Researchers. Latin America.

\footnotetext{
${ }^{1}$ Universidad Nacional de Tres de Febrero - Buenos Aires, Argentina.

E-mail: cesargeronimotello@yahoo.com.ar

DOI: $10.1590 / E S 0101-73302019205680$
} 


\title{
NOTAS HISTÓRICAS E EPISTEMOLÓGICAS SOBRE O CAMPO DA POLÍTICA EDUCATIVA NA AMÉRICA LATINA
}

\begin{abstract}
RESUMO: Neste trabalho se apresentam algumas notas históricas e epistemológicas sobre o campo da política educativa na América Latina, ressaltando o enfoque das epistemologias da política educativa como um modo de análise das investigações que os pesquisadores realizam no campo. Este artigo tem a intençấo de repensar o campo das políticas educativas das transformaçóes e a crise das ciências sociais e políticas diante da necessidade de compreender e nomear a complexa realidade socioeducativa atual.
\end{abstract}

Palavras-chave: Política educativa. Epistemologia. Investigação educativa. Pesquisadores. América Latina.

\section{Presentación}

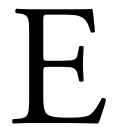

ste trabajo tiene por objeto describir algunas notas epistemológicas e históricas del campo en Latinoamérica. Abordando, así, la categoría teórica de las Epistemologías de la política Educativa (TELLO, 2009; 2011) como un modo de pensar y reflexionar sobre la producción del conocimiento del campo de la política educativa y a partir de la cual hemos construido el Enfoque de las epistemologías de la política educativa (EEPE) (TELLO, 2012) como un enfoque analítico para el campo de producción de conocimientos en el campo.

Debemos realizar cuatro seńalamientos para comenzar a caracterizar las epistemologías de la política educativa, estos pueden considerarse como no-novedosos, pero necesarios para el desarrollo conceptual de este trabajo.

Los dos primeros se vinculan a la posibilidad de conocer.

Nos referimos en primer término a la no neutralidad de la investigación y de la producción del conocimiento (HABERMAS, 1981; 1984; LEBLOND, 1975; SOUSA SANTOS, 2006; 2009; entre otros) en nuestro caso, en política educativa y, en segundo término a la necesidad de establecer el lugar en que se posiciona el investigador para llevar a cabo su proceso de investigación y desde qué perspectiva realiza su análisis y obtiene determinados resultados (TELLO, 2012; TELLO; MAINARDES, 2012).

En este sentido adherimos al pensamiento de Sousa Santos (2006) cuando denuncia que la producción del conocimiento desde una perspectiva positivista es tomada como independiente de la cultura. Pues, podemos buscar algún tipo de objetividad relativa, dado que existen algunas metodologías que nos permiten hacerlo, pero nunca como investigadores en política educativa podemos situarnos en o desde la neutralidad. Es necesario considerar el postula- 
do de Max Weber sobre la "neutralidad valorativa" que suponía, al igual que el positivismo, la falacia de una ciencia "libre de valores" y una radical separación entre sujeto cognoscente y objeto conocible. Pues, el no establecimiento de la posición del investigador lo dejaría muy cerca de una posición positivista (que considera la neutralidad de las ciencias y del conocimiento producido) o del desconocimiento epistemológico para llevar a cabo una investigación. (En muchas ocasiones el investigador lo hace porque considera que "está claro" desde dónde se habla, y así sucede que el investigador no revisa ni explicita sus postulados epistemológicos en el proceso de investigación).

Para comenzar a desplegar las notas epistemológicas e históricas que aquí nos proponemos es imprescindible plantear los otros dos señalamientos que se relacionan con el campo teórico de la política educativa.

El primero: debemos señalar la distinción entre Estudios sobre la Política Educativa, o lo que denominamos campo teórico, y las Políticas Educativas ${ }^{1}$ (aquellas que hacen referencia específica a la gestión, toma de decisiones y acción política). Max Weber (1991, p. 83) en El político y el científico, sostuvo con énfasis la clara diferenciación que distingue a "las tomas de posición política" (Políticas educativas en plural) del "análisis científico de los fenómenos políticos" (Política Educativa en singular).

El EEPE se centra en el meta-análisis del proceso de investigación del campo, es decir el modo en que se plantean, diseñan y se llevan a cabo las investigaciones en política educativa por parte del sujeto que investiga, con sus supuestos teóricos y perspectivas.

Es decir, se concentra en la política educativa como campo teórico. Sin embargo, podría emplearse también sobre las politicas educativas en tanto los fundamentos ya no tanto epistemológicos sino ontológicos. En tanto concebimos la ontología como el análisis de los fines y horizontes en términos éticos.

Así cabe la pregunta ¿cuál es la base onto-epistemológica de las políticas educativas? Por ejemplo de un país o una región. Cuáles son las finalidades que se observan en esas políticas educativas con los programas implementados.

Estas elucubraciones nos conducen a tener en cuenta la advertencia de Ana Vitar (2006, p. 26) cuando afirma: "No debemos confundir los procesos políticos y sociales con las herramientas para pensarlos".

El segundo señalamiento: nos referimos a epistemologías, en plural, dado que existen diversas concepciones teóricas para la investigación en política educativa. Existen múltiples epistemologías de la política educativa, sin embargo se debe tener en cuenta que en la producción de conocimiento de cada investigador se tendría que poder observar una epistemología coherente y sólida con los tres componentes que conforman parte del EEPE: Posicionamiento epistemológico, Perspectiva Epistemológica y Enfoque Epistemetodológico. Esto lo veremos detenidamente más adelante. 


\section{Algunas consideraciones actuales sobre las ciencias sociales y las ciencias políticas}

En 1958 Braudel (1987) escribía "Hay una crisis de las ciencias del hombre...”, esta aseveración se mantuvo y expresó de diversos modos en los últimos años. Existen múltiples razones por las cuales podríamos elucubrar que las ciencias sociales ingresaron en un período de crisis, pero principalmente podemos considerar que el mundo social para denominarlo desde un lugar amplio ha sufrido vertiginosos cambios y transformaciones aceleradas, y así las diversas categorías de las ciencias sociales fueron quedando al margen de la potencialidad de miradas que le permitan comprender la realidad social. Estamos atravesando una etapa de profundos cambios sociales, con realidades cada vez más complejas de ser abordadas por las estructuras conceptuales convencionales, lo cual conlleva a que la realidad de los fenómenos que se nos presentan a los investigadores en ciencias sociales carezca de referentes conceptuales y viceversa, las estructuras y categorías conceptuales con las que nombrábamos de algún modo lo que acontecía socialmente carecen de potencialidad explicativa. En este sentido Wallerstein (1999) plantea que las ciencias sociales tienen grandes limitaciones en el estudio de las realidades sociales por lo cual habría que pensar nuevos fundamentos epistemológicos, para esto propone un desafío: "impensar las ciencias sociales" que se opone a la categoría de re pensarla, dado que re pensarlas se convertiría en una barrera epistémica para la creación de nuevas epistemologías.

En el mismo sentido, las ciencias políticas se enfrentan a interrogantes similares, por ejemplo, siguiendo los postulados de Giovanni Sartori (2004) en un reconocido artículo denominado “¡Hacia dónde va la ciencia política?”, César Cansino en el año 2006 (y luego en el 2008) anuncia el adiós a la ciencia como la crónica de una muerte anunciada con muchos debates a su alrededor ${ }^{2}$. Explicando que la ciencia política:

terminó alejándose del pensamiento y la reflexión, hasta hacer de esta ciencia un elefante blanco gigantesco, repleto de datos, pero sin ideas, ni sustancia, atrapada en saberes inútiles para aproximarse a la complejidad del mundo (CANSINO, 2006, p. 13).

En el mismo eje de reflexión las ciencias sociales se encuentran debilitadas en la reflexión disciplinar para la realidad Latinoamericana (DE SIERRA et al., 2006) lo cual requiere en la perspectiva de Atilio Borón (2006, p. 16) "una minuciosa discusión sobre algunos de los principales problemas que plantea la necesaria reconstrucción de las ciencias sociales sobre nuevas bases epistemológicas".

Ciertamente existe una crisis de los campos de las ciencias políticas y las ciencias sociales, considerando que las actuales matrices epistemológicas de los campos no se sostienen con solidez ante la realidad que debe investigar para 
comprender, esto se debe a que las estructuras y categorías conceptuales se han desplegado como un modo normativo y hasta meramente procedimental de llevar a cabo investigaciones en el campo de las políticas educativas.

La realidad de las ciencias sociales y en particular de la política educativa requiere una transformación epistemológica que permita construir y desplegar nuevas epistemologías para repensar la política educativa, a partir del atrevimiento científico que, de algún modo, resquebraje y sacuda las perspectivas epistemológicas actuales, lo cual permitirá - a partir de esa deconstrucción — un espacio para iniciar un nuevo camino epistemológico en Latinoamérica. Sin dudas que varios pensadores de diversas latitudes del mundo están dando cumpliendo al legado de Bachelard (1994, p. 19) cuando afirmar que "solo existe un modo de hacer avanzar la ciencia; es la de atacar la ciencia ya constituida, o sea cambiar su constitución".

Por tanto, no se trata solo de contraponer una teoría a otra teoría, la cuestión principal es desplegar conceptos y categorías teórico-analíticas potentes para el análisis de las realidades sociopolíticas educativas actuales y complejas.

\section{Notas históricas}

Las ciencias sociales - o el pensamiento en ciencias sociales - se podría ubicar en Latinoamérica con el surgimiento de los Estados-Nación en Latinoamérica ${ }^{3}$. Por su parte las ciencias políticas deben entenderse en el marco del desarrollo de las ciencias sociales; el nacimiento de la ciencia política moderna se produce a través de la separación de los estudios políticos respecto de la matriz tradicional del derecho. El año 1948 marca el hito fundacional en la ciencia política: a pedido de la UNESCO, se reúnen en París expertos y estudiosos con la finalidad de tratar de redefinir y acotar su objeto de estudio. Las deliberaciones concluyen con la confección de la célebre "Lista Tipo" influencia del pensamiento anglosajón. Si bien ella constituye tan solo una mera enumeración pragmática de temas, sigue siendo a pesar de los sesenta años de su elaboración un eje de referencia para la elaboración de marcos teóricos y la confección de currículas universitarias en relación a las ciencias políticas incluyendo el área curricular de la política educativa actual en las carreras de pedagogía y ciencias de la educación. En este sentido y siguiendo el pensamiento de Kuhn (1991) esta cuestión se convierte epistemológicamente en una gran preocupación dado que el historicismo radical de la ciencia sugiere una concepción de continuidad con la contemporaneidad esto es: quedar "atado" al pensamiento vigente de la época sin posibilidades de creación de nuevos referenciales

Es en este período [finales de 1940, comienzos de 1950] que se comienzan a desarrollar en distintas universidades latinoamericanas las ciencias políticas como campo de estudio con "pretensión científica", y casi exclusivamente desde el enfoque jurídico-institucionalista (BARRIENTOS DEL MONTE, 2009). Esto se 
produce considerando que, finalizada la Segunda Guerra Mundial, se desarrollaron políticas de bienestar que se centraron en cuestiones tales como la salud y la educación. De este modo se puede observar que comienza a emerger un nuevo modo de concebir las políticas públicas en Latinoamérica (GOROSTIAGA; TELLO; ISOLA, 2012). Con el despliegue de las políticas de bienestar, los organismos de gobierno apelaron a los investigadores en ciencias sociales en busca de soluciones a los problemas que suponía la planificación de las actividades estatales, incluyendo las relacionadas con el crecimiento y reforma de los sistemas educativos.

En este marco, cuanto comenzaba a desarrollarse la ciencia política como campo de estudio, mayor era el volumen y la variedad de los temas a estudiar. Lo cual se convirtió en una dificultad en ese momento - lo que es común en los campos emergentes del conocimiento - debido al esfuerzo que suponía el establecer una lógica coherente en el aumento de cantidad de temáticas que el campo debía abordar, pero ese esfuerzo de apertura del campo (BOURDIEU, 2000) daría lugar de algún modo a la política educativa, como un espacio insipiente que se desprendía - desde la perspectiva histórica de ese momento - como subdisciplina de la ciencia política.

\section{Surgimiento del campo de las políticas educativas: la institucionalización}

Dotadas de las características principales las ciencias políticas surgían en la década de 1950 la política educativa como campo teórico, con una visión fuertemente centrada en la legislación y en algunos casos en Educación comparada. Esta última no poseía el desarrollo epistemológico actual sino que básicamente consistía en establecer ejes de comparación legislativos de diversos países y de la estructura del sistema educativo, entre otros temas de comparación lineal y casi descontextualizada. Pero sin duda respondía a la matriz analítica de las ciencias políticas en Latinoamérica con el enfoque jurídico-institucionalista.

En esta línea de análisis consideramos que el estudio del surgimiento del campo supone algún acuerdo respecto a aquello que se valora en el campo, "los que participan en la lucha contribuyen a reproducir el juego, al contribuir, de manera más o menos completa según los campos, a producir la creencia en el valor de lo que está en juego" (BOURDIEU, 2003, p. 122).

Esta conceptualización bourdieana permite señalar la existencia de un campo como el conjunto de prácticas, sentidos, mecanismos regulatorios, etc. que se estructuran en torno a las actividades que desarrollan las instituciones, en el caso de este trabajo, particularmente las instituciones universitarias y los actores que en ella participan (STREMEL, 2017). En esta década se comienza a observar en Argentina, Brasil, Chile, México, Colombia, entre otros países de Latinoamérica, algunos procesos de "institucionalización" de la política educativa como campo 
y que se despliega a través de la creación de cátedras de Políticas Educativas o espacios curriculares con denominaciones similares. Teniendo en cuenta que no nos referimos al inicio de la investigación en política educativa o a reflexiones políticas sobre la educación, dado que estas, siguiendo la consideración del surgimiento de las ciencias sociales esgrimido anteriormente por Castro-Gómez (2000) podrían ubicarse en el propio surgimiento de los Estados en Latinoamérica, sino considerando la conceptualización de "institucionalización" en términos de Gómez Campo y Tenti Fanfani (1989) cuando explican que los procesos por los cuales ciertas prácticas se recortan como específicas, sea con la creación de una cátedra, una carrera, un centro de estudios o un departamento en el ámbito universitario dan muestra del proceso histórico y la presencia real de un espacio institucional que permite la circulación y producción del conocimiento.

\section{El marco del proceso de institucionalización del campo}

La institucionalización del campo de las políticas educativas con el surgimiento de cátedras de Política Educativa se debe comprender en el marco del surgimiento de las "nuevas" carreras de ciencias de la educación en Latinoamérica (GOROSTIAGA; TELLO; ISOLA, 2012) con cierta sistematización para la enseñanza y la investigación, en sus diversas versiones: Política Educacional, Política y Legislación escolar, Política y educación comparada y Política y organización escolar. Considerando el intercambio de nombres para las diversas cátedras entre 1950 y 1970.

Otro de los cambios que impacta sobre las "nuevas" carreras de ciencias de la educación y que fortalecieron la presencia y continuidad de las cátedras en las currículas universitarias se asocian al surgimiento de la corriente del desarrollismo latinoamericano en tanto los perfiles y funciones de los graduados en ciencias de la educación, considerando que en esta corriente se requería desde una perspectiva técnica: ejecutores de políticas educativas, analistas de la legislación escolar, planificadores educativos, técnicos, y analistas estadísticos en educación entra otras funciones y, desde una perspectiva teórica: la vinculación con los "países desarrollados", la formación de recursos humanos y una perspectiva que permitiera identificar -en términos de ese período- las dificultades del subdesarrollo latinoamericano. Según Rama (1984, p. 11), estas nuevas orientaciones tuvieron su principal origen "en los centros especializados en problemas de planificación económica, con una rápida difusión al contar con el apoyo de la planificación educativa”.

En el marco de esta reorientación teórica y epistemológica, desde fines de los 50 y hasta comienzo de los 70, las cátedras de política educativa comienzan a surgir con una preocupación del "valor de lo que está en juego": el análisis teórico y desarrollo de competencias de los graduados en pedagogía o ciencias de la educación para la planificación, la comprensión de la legislación escolar, políticas de universalización de la educación primaria y secundaria. En fin, la investigación 
educativa, y así el campo de las políticas educativas, recibieron en la región un fuerte impulso como consecuencia del establecimiento de carreras universitarias en educación. Los estudios conducidos en esa época "consistieron fundamentalmente en análisis descriptivos o estadísticos, para determinar sectores de población donde había que desarrollar la educación primaria o secundaria" (NAZIF; ROJAS FIGUEROA, 1997, p. 3).

Así se puede afirmar que el proceso de constitución del campo de la política educativa como campo teórico en Latinoamérica transcurrió entre 1950 y 1970.

\section{Las etapas históricas del campo}

Desde su surgimiento, el campo de la política educativa atravesó vaivenes de corrientes teóricas, filosóficas e interrupciones de política democrática, de ideas y conceptos que la estructuran y que se han transformado con los cambios científicos y sociales de la propia realidad educativa, experimentando de este modo numerosos cambios que presentan tanto rupturas como continuidades. Es por esto que creemos que la situación actual del campo se comprenderá de forma más acabada si se la contempla desde su proceso histórico. Con esto queremos decir, las epistemologías de la política educativa que en la actualidad se pueden crear, serán distintas a la del surgimiento del campo y en consonancia, a las que se puedan plantear en los próximos veinte ańos, porque las epistemologías se conforman en los contextos culturales, en una fuente de ideas, en un determinado tipo de relaciones teóricas, como hemos advertido anteriormente en términos kunhianos. Y de este modo afirmamos gratamente que las epistemologías de la política educativa que se nos presentan no serán las de antaño ni las venideras, allí es donde debemos estar atentos a que las epistemologías de la política educativa cumplan con su condición dinámica: captar, nombrar, comprender y/o describir la realidad socio-educativa. Por tanto preguntas que se suponían "contestadas" acabadamente en determinado período es necesario volver a estudiarlas a luz de los nuevos interrogantes que se redimensionan ante la realidad y el despliegue teórico que se va desarrollando en el campo.

Por eso es necesario, al menos mencionar, lo que consideramos algunas notas históricas del campo que denominaremos etapas a modo provisorio, no intentaremos realizar una estructuración cronológica rigurosa. Dado que toda cronología que intente desplegar la historia del campo de la política educativa puede adolecer de imprecisión, por el sólo hecho de entender la historia como un proceso dinámico, superpuesto e hilvanado.

La primera etapa que denominamos como "la política educativa y el enfoque jurídico-institucionalista" se caracterizó porque su objeto de estudio se centraba principalmente en el análisis de la normativa legal, en este sentido y to- 
mando la perspectiva de Bobbio (1989) llevó a que los abogados y especialistas en jurisprudencia fueran durante un largo período, hasta 1960 en Latinoamérica, los responsables autorizados de reflexionar sobre el Estado y las políticas educativas. Esto se observa claramente en las cátedras de política y legislación escolar que se incluyen en los planes de estudios universitarios. La segunda etapa, que ubicamos en los inicios de la década de 1960 y que denominamos como "la política educativa en la perspectiva de la planificación desarrollista" tuvo su base en cuanto a perspectiva teórica en conjunto con el proceso de institucionalización y fortalecimiento de las ciencias en la región a través de la creación de oficinas estatales, institutos de investigación y organismos regionales (CALDERÓN; PROVOSTE, 1992; DE SIERRA et al., 2006). Es en estas décadas que podríamos hablar del período de protagonismo de la Economía, la sociología y la ciencia política en los ámbitos académicos dado que se comienza a desarrollar una demanda por parte del Estado, como hemos mencionado anteriormente. Es así que en la década de 1960 se observa que el campo de la política educativa está fuertemente vinculada a la teoría de la planificación y el planeamiento educativo, con el auge del desarrollismo y la teoría del capital humano en Latinoamérica (SUÁSNABAR, 2009). Es el período de la política educativa que se desarrolla desde el enfoque de la planificación.

Durante la década del sesenta predominaron las corrientes denominadas del "desarrollo" y del "capital humano", que ponían el énfasis sobre otros aspectos de los sistemas educacionales. La mirada se dirigió hacia cómo crecen, cómo se distribuyen social y regionalmente, cuál es su eficiencia interna (rendimiento cuantitativo) y cuál es su eficacia (adecuación en número y tipo de egresados a las necesidades de mano de obra). Se desarrolló la Sociología de la Educación bajo el paradigma funcionalista y la Economía de la Educación bajo el paradigma del capital humano (PAVIGLANITI, 1993, p. 9).

La tercera etapa, hacia fines de la década de 1960 y durante la década de 1970 que denominamos "la política educativa y la perspectiva crítica", es la del surgimiento de las miradas y enfoques críticos-reproductivistas que comenzaban a cuestionar el funcionamiento de los sistemas educativos nacionales, dando lugar a los estudios de etnografía educativa dentro de las políticas educativas, al desarrollo de la sociología política, y a perspectivas neo-marxistas y pos-estructuralistas entre otras.

Su contribución principal consistió en quebrar la mirada optimista, de orden lineal y progresivo de crecimiento, construida por las clases dominantes en las décadas del cincuenta y del sesenta. Además, pusieron de relieve las formas y los mecanismos de la desigualdad social que están incorporados en los sistemas Educativos, sacaron a la luz sus conexiones con las diferencias culturales, con la reproducción de la mano de obra y con la consolidación de las relaciones sociales y políticas (PAVIGLIANITI, 1993, p. 11). 
La década de 1970 encuentra a los países de Latinoamérica cruzados por dictaduras feroces que paralizaron el desarrollo del campo, particularmente de las últimas concepciones neo-marxistas. Comienzan a desarrollarse enfoques como la "educación como restauradora del orden", del "orden natural", del "personalismo" y del "estudio de los agentes de la educación” (PUIGGRÓS, 1986).

A medida que fueron finalizando los gobiernos dictatoriales en Latinoamérica comenzó a emerger el optimismo pedagógico abriendo los caminos de investigación hacia el paradigma de educación y democracia (SUÁSNABAR, 2009) expresado generalmente en la perspectiva de educación y sociedad. Así, la cuarta etapa se da con la instauración de las democracias latinoamericanas, pasando por los enfoques de "Política y administración de la educación" y luego influenciada por el neoliberalismo a "política y gestión de la educación" en sus dos vertientes, la vinculada al managment y la que lo hace desde una perspectiva humanista o antihegémonica (MINANA BLASCO; RODRÍGUEZ, 2003).

Cuando se introdujo la temática de gestión educativa en Latinoamérica se la asociaba, desde las perspectivas críticas, a una visión liberal que intentaba evaluar el fenómeno educativo en términos de costo-beneficio. Ahora bien, de modo simultáneo emerge la categoría temática de gestión educativa, como campo disciplinar, que data de los años sesenta en Estados Unidos, de los años setenta en el Reino Unido y de los años ochenta en América Latina (CASASSUS, 2000).

\section{La política educativa como campo teórico y las epistemologías de la política educativa}

Definimos el campo de la política educativa como campo teórico, homologable al campo académico en términos de Bourdieu (2000) y Bourdieu, Chamboredon e Passeron (2003), en tanto desde ese campo se produce conocimiento (investigación) se genera circulación de conocimiento (formación académica) y se desarrolla un uso o aplicación de ese conocimiento (la profesión), considerando que no necesariamente estos tres espacios son consecutivos.

Debemos considerar que el objeto de estudio de la política educativa es la propia realidad socio-educativa en sus múltiples dimensiones, sin dudas que esta afirmación puede ser atribuible a la sociología de la educación, la pedagogía, la historia de la educación, entre otras. Ahora bien, estos campos teóricos construyen e intentan comprender la realidad educativa desde diversos ángulos de análisis. Para el caso de la política educativa, consideramos que existen ejes que le son propios, aunque no exclusivo, y que confluyen en su objeto de estudio. Entre los principales ejes podemos mencionar: el Estado, la jurisprudencia, la micropolítica, la sociología política, el discurso político, los debates políticos, la gobernabilidad educativa, entre otros. 
En Latinoamérica, comenzar a caracterizar las epistemologías de la política educativa puede venir en auxilio del status epistemológico del campo. Con esto no hacemos referencia a un núcleo teórico perteneciente a la política educativa, ni a definir su carácter de disciplina o ciencia ${ }^{5}$, sino a varios núcleos teóricos. Este es uno de los principales aportes que consideramos que puede realizar este trabajo: contribuir al fortalecimiento epistemológico del campo teórico, sin tener como horizonte la distinción entre ciencia, disciplina o subdisciplina. Por el contrario el fortalecimiento epistemológico del campo teórico se da en la medida que asume su multidisciplinariedad y complejidad interdisciplinaria en el desarrollo para la investigación en política educativa, que se manifiesta en su diversidad de Posicionamientos Epistemológicos, Perspectivas epistemológicas y Enfoques epistemetodológicos, así, afirmamos que las epistemologías de la política educativa se conforman cuando se puede observar en la investigación un Posicionamientos Epistemológicos, Perspectivas epistemológicas y Enfoques epistemetodológicos que se constituyen en los componentes del EEPE.

\section{Ahora bien, ¿qué entendemos por cada una de estas categorías?}

Hemos desarrollado estas categorías - en tanto enfoque - como el conjunto de tres componentes: la posición epistemológica, la perspectiva epistemológica y la perspectiva epistemetodológica. Considerando el EEPE como un modo de reflexionar sobre la investigación en el campo. Y entendiendo que existen diversas "Epistemologías" en plural dado que existen diversos posicionamientos y perspectivas epistemológicas para desarrollar y llevar a cabo una investigación en política educativa, esto es: existen diversas epistemologías de la política educativa ${ }^{6}$.

Entendemos por Perspectiva Epistemológica para el caso de las investigaciones en política educativa la cosmovisión que el investigador asume para guiar la investigación, de algún modo lo que Glaser y Strauss (1967) han denominado la Teoría General. Debemos considerar que no existe una "cabeza vacía" en términos epistemológicos, sin embargo existen "cabezas abiertas" ${ }^{\text {en búsqueda }}$ de resultados de investigación. Ejemplo de perspectivas podrían ser el marxismo, neo-marxismo, estructuralismo, pos-estructuralismo, existencialismo, humanismo, positivismo y pluralismo.

El Posicionamiento Epistemológico se desprende desde la propia Perspectiva Epistemológica o debería desprenderse en una investigación coherente y consistente. El Posicionamiento epistemológico, esto es la Teoría Sustantiva (GLASER; STRAUSS, 1967) vinculada particularmente al campo de estudio, es decir a las corrientes teóricas propias del campo, considerando que las teorías sustantiva son aquellas que guardan una relación directa con el contenido empírico y teórico de los datos de la investigación. Este es el eje y la nervadura del EEPE, dado que es aquí donde se pone en juego la presencia de la cosmovisión del investigador. 
Entendiendo la cosmovisión como "un conjunto de presuposiciones (o premisas) que sostenemos acerca de la constitución básica de nuestro mundo" (SIRE, 2004, p. 17). Esto es, no solo modos de leer la realidad, sino los modos de construirla, en términos de reflexividad epistemológica. En este sentido el posicionamiento epistemológico se convierte en el posicionamiento político del investigador. Podemos mencionar los posicionamientos neoinstitucionalistas, institucionalista, clásico o jurídico-legal, constructivismo político, de la complejidad, eclecticismo, posmoderno, posmodernista, hiperglobalista, escéptico, neoliberal, enfoque transformador, funcionalista, crítico, crítico radical, crítico-analítico, teóricos de la resistencia, crítico-reproductivista, humanista, economicista, etc.

El posicionamiento epistemológico reposa sobre la selección que realiza el investigador en cuanto la perspectiva epistemológica con la que desarrollará su investigación.

En fin, tanto la perspectiva como el posicionamiento epistemológico no emergen de una "cabeza vacía" por el contrario poseen sustrato y sustento. La dificultad se observa cuando el investigador en políticas educacionales no puede nombrar lo que le está aconteciendo como perspectiva o posicionamiento epistemológico e intenta construir su investigación desde la pseudoneutralidad.

En un estudio reciente (TELLO; MAINARDES, 2012) hemos observado que existen investigaciones con una clara posición epistemológica del investigador respecto a la construcción epistémica de la investigación en política educativa. Sin embargo, hemos observado que en muchas investigaciones sobre política educativa no se deja claro "desde dónde se investiga", en este sentido argumentamos que los investigadores poseen una posición epistemológica que tiene como base la idea que el desarrollo de una investigación reposa sobre una cierta visión del mundo y así asume inevitablemente una perspectiva y un posicionamiento epistemológico (MARTINET, 1990).

Finalmente, el componente que hemos denominado Enfoque Epistemetodológico. Con el cual nos referimos al momento metodológico donde el investigador opta por una u otra metodología. No consideramos a los 'enfoques metodológicos' como meros instrumentos sea de recopilación, sea de análisis de la información. Sino como el "método del logos", esto es el modo de pensar el logos. Por tal razón preferimos hablar de epistemetodología, categoría en la que confluyen la presentación de método y la posición epistemológica del investigador. Consideramos que un enfoque metodológico posee una epistemología, pero a los efectos de la distinción de uso común, preferimos este último término: epistemetodología. No lo hacemos por una cuestión de mera sofisticación en los conceptos, sino más bien de precisión (TELLO, 2011). Es decir, debemos tener en cuenta que la metodología se convierte en una epistemetodología en la medida que está enhebrada a los otros componentes: Perspectiva epistemológica y Posición Epistemológica. Por sí sola y, por ejemplo a los efectos de la enseñanza son sólo metodologías, con mayor precisión conceptual nos podemos referir a la 
metodología como técnicas metodológicas. Técnicas que muchas veces se enseñan erróneamente como epistemologías, y en otros casos, como dijimos, sin sustento epistemológico. Bourdieu (2000, p. 62) es agudamente irónico respecto de esta predominancia explicando que se la toma como "serie de recetas o de preceptos que hay que respetar no para conocer el objeto sino para ser reconocido como conocedor del objeto". Por su parte, De Miguel (2000) afirma:

En la práctica es habitual especificar los planteamientos metodológicos con que se aborda la evaluación de un programa pero rara vez se hace mención alguna a las concepciones sobre la naturaleza de la realidad que es objeto de análisis (bases ontológicas) o sobre los fundamentos para la construcción del conocimiento científico (bases epistemológicas) (apud SANDÍN, 2003, p. 43).

Teniendo en cuenta esta advertencia es necesario que la metodología en términos de investigación se convierta en epistemetodología, en tanto constituya un componente consistente de la red de los tres componentes que conforma el EEPE. De este modo una investigación en política educativa puede ser analizada tratando de identificar estos tres componentes, los cuáles nos permiten también comprender la complejidad de una investigación propia del campo, esto es, no encontraremos sólo uno de los componentes en el tejido de la investigación sino que existirá una Perspectiva epistemológica, un Posicionamiento Epistemológico y un Enfoque Epistemetodológico, con mayor o menor coherencia y consistencia entre sí. En síntesis, la cuestión que presentamos no es si la investigación y la proyección social que realizan las ciencias humanas y sociales contienen o no valores e ideología, sino la necesidad de indagar qué valores se encuentran en cada proceso y situación, cuál es su papel y cómo se podrán, y deberán — desde las diferentes visiones de realidad - hacer explícitas en el proceso de investigación. Un principio para tener en cuenta es que no se puede eliminar la subjetividad y los valores de la ciencia y de sus usos técnicos; habría, en cambio, que esforzarse en hacerlos explícitos, para observar su aportación al resultado final de la actividad de investigación en política educativa. Con esta orientación, el investigador asume mayor libertad de elección de un curso de acción o de un método determinado pero, paralelamente, mayor es la responsabilidad (personal y profesional) por las consecuencias de su proceder y por el valor social de los resultados de sus investigaciones, en tanto posición política: “¿Hasta qué punto ese conocimiento contribuye o no a la posibilidad de construir una sociedad más democrática y más equitativa?" (LANDER, 2000, p. 54).

Podríamos pensar en la posibilidad de definir algunos rasgos de las epistemologías de la política educativa pero nunca considerar que se podría definir la "teoría de la política educativa" entendida esta última como una reducción epistemológica, dado que los planteos epistemológicos actuales no requieren de la "demarcación" disciplinar, lo cual responde más bien a estudios y concepciones clásicas desde la epistemología que distinguía por ejemplo: Ciencia-Pseudocien- 
cia-Disciplinas con un objeto de estudio delimitado y metodología propia, entrando en el falso camino del cientificismo; por el contrario, asumimos en el sentido de Wallerstein (2004), una postura a favor de la ciencia y el conocimiento y en contra del cientificismo.

De este modo, consideramos el campo de la política educativa como un espacio reticular, en tanto se define un campo de conocimiento desde la epistemología contemporánea como la posibilidad de nuevas construcciones conceptuales que permiten la interacción múltiple y recíproca entre distintos campos de conocimiento. En términos de Gianella (2006, p. 79): "El retículo tiene una estructura doblemente compleja, ya que en la red se admiten integrantes que a su vez son redes". Y es allí donde se produce lo que mencionábamos anteriormente: una vinculación con categorías de la ciencia política, la sociología de la educación, la pedagogía y, donde la clave está en los múltiples objetos de estudio que construye la política educativa.

\section{Consideraciones finales: perspectiva epistemológica de la política educativa como campo teórico}

La epistemología, en su concepción actual, suele entenderse como Teoría de la Ciencia. Ahora bien, desde nuestra perspectiva es imprescindible comprender las epistemologías de la política educativa no como ciencia, sino como enfoque, método y reflexión sistematizada del análisis de la producción de conocimiento en el campo. Una epistemología que asuma la connotación social, política y cultural del conocimiento y la investigación. Sería errónea o simplificadora la concepción meramente instrumental que muchas veces se le otorga a la epistemología, como la que posibilita las miradas meta teóricas de un campo. Quedarnos en esa posición, insisto, sería reducir la potencialidad y fertilidad de la epistemología para el análisis de campos de conocimiento, en nuestro caso el campo de la política educativa como campo teórico. Concibiendo el Campo Teórico como la posibilidad de observar y transformar la realidad y allí, otra característica de las epistemologías de la política educativa: entiéndase transformación de la realidad en tanto la misma es observada y analizada. No con un objetivo instrumental, o de intervención sobre la misma con una connotación meramente empírica, de datos, o falsas resoluciones de problemas de la realidad.

Consideramos que la investigación en política educativa requiere una transformación epistemológica, como se reclama desde las ciencias sociales y las ciencias políticas, una transformación epistemológica que sea capaz de desafiar las grandes verdades establecidas y desde la investigación repensar la política educativa.

En este sentido, debemos considerar el planteo de Stephen Ball (2006) cuando se refiere a la investigación en políticas educativas señalando las estructuras de una investigación, por un lado la que el autor denomina "epistemología de su- 
perficie" como la relación que se establece entre conceptualizaciones, conducción, diseño y desarrollo de una investigación. Es decir allí se encuentra un modo de hacer epistemología, pero no puede dejar de tenerse en cuenta para la solidez y consistencia de la misma la "epistemología profunda", esto es las formas de explicación: lo que hemos denominado en este trabajo como el EEPE.

Las consideraciones vertidas por Stephen Ball (2006) son tomadas de un modo erróneo como pasos sucesivos, nos referimos a la falsa concepción del Paso I y del Paso II que se presentan en una investigación. Entendiendo el Paso I como el propio diseño: el recorte de un objeto de estudio, la selección empírica, la propuesta de objetivos de investigación como si estos pasos estuvieran "libres" de un posicionamiento, el Paso I no es una tarea meramente técnica o de "superficie" y, si así se lo tomase la dificultad se encontraría en cuanto que muchas investigaciones se llevan a cabo desde la "epistemología de superficie", es decir muchas investigaciones en política educativa son investigaciones que sólo desarrollan prolijas cuestiones técnicas. Y es aquí donde debemos reflexionar acerca de que la metodología no es solo "una metodología", sino epistemetodología en términos de la epistemología de la política educativa. En términos de Bourdieu (2000) es clave conocer desde "donde se habla", desde que lugar se posiciona el investigador.

Sin dudas que asistimos a un nuevo modo de comprender la realidad, cuyos efectos están cambiando radicalmente nuestra manera de conocer el mundo y, como si lo anterior no fuera suficiente, de relacionarnos con él. En este sentido debemos considerar que el campo de la política educativa cuyo continente son las ciencias sociales requiere de un nuevo espacio de reflexión epistemológico luego de la "tormenta neoliberal" (TORRES, 2008).

El enfoque de las epistemologías de la política educativa intenta de algún modo proponer una mirada que contemple la realidad social que nos convoca a los investigadores desde la profundidad de la epistemología; una epistemología que debe ser parte de la realidad y contribuir a ella.

Desde mi perspectiva, las epistemologías de la política educativa contribuirán a que la claridad teórica y la reflexividad en torno a las propias producciones de políticas educativas generarían mayor precisión en los diálogos entre investigadores y en este sentido un fortalecimiento del campo. Teniendo en cuenta que existirán otras epistemologías: la de la opresión, las hegemónicas o simplemente las de adhesión a sistemas políticos. Esto es en el sentido de Gewirtz (2007, p. 7) llevar a cabo una "reflexividad ética sobre el trabajo de investigación", reflexionar sobre las posibles consecuencias éticas de la investigación, de qué forma ciertos trabajos legitiman determinadas epistemologías y no otras.

Pero quizá considerando las etapas históricas desarrolladas brevemente en este trabajo, conjuntamente con el desafío que nos propone las ciencias sociales y políticas concentremos esfuerzos para "impensar" las políticas educativas, y así contribuir a las epistemologías de la política educativa. Si logramos avanzar en 
este camino, probablemente también, podamos pensar en una epistemología de la política educativa latinoamericana (TELLO, 2010). En la cual la investigación en políticas educativas contribuya a fortalecer y desarrollar la justicia educativa y la consolidación democrática en nuestra América.

\section{Notas}

1. No tomamos aquí la confusa distinción anglosajona entre politics y policy. Dado que esta distinción es ambigua al intentar traducirla al español. Como afirma Cox (2006, p. 3), "no se puede recuperar el sentido del segundo término si no es especificando políticas sectoriales"

2. La fuerte crítica a la ciencia política, que tiene pretensiones hegemónicas, provocó la discusión, la controversia y la polémica. Prueba de ello son los trabajos de Negretto (2004); Colomer (2004); Laitin (2004); Zolo (2006); Oxhron (2006); Molina (2006); Covarrubias (2006) y Lucca (2008) que, de una u otra forma, presentan posiciones en el marco de la polémica.

3. En este sentido Santiago Castro-Gómez (2000, p. 149) explica que el surgimiento de las ciencias sociales no es un fenómeno posterior a los modos de organización política de los surgentes Estados-Nacional en Latinoamérica "sino constitutivo de los mismos". Se requería de "lineamientos científicos" sobre la realidad para fundamentar los modos de gobernar. Es decir: "Sin el concurso de las ciencias sociales, el Estado moderno no se hallaría en la capacidad de ejercer control sobre la vida de las personas, definir metas colectivas a largo y a corto plazo, ni de construir y asignar a los ciudadanos una "identidad" cultural" (CASTRO-GÓMEZ, 2000, p. 147).

4. Los distintos temas propuestos quedan agrupados en cuatro secciones: I. Teoría política: a) Teoría política. b) Historia de las ideas políticas; II. Instituciones políticas: a) Constitución. b) Gobierno central. c) Gobierno regional y local. d) Administración pública. e) Funciones económicas y sociales del gobierno. f) Instituciones políticas comparadas; III. Partidos, grupos y opinión pública: a) Partido político. b) Grupos y asociaciones. c) Participación del ciudadano en el Gobierno y la Administración. d) Opinión pública; IV. Relaciones internacionales: a) Política internacional. b) Organización y administración internacional (UNESCO, 1948).

5. No adherimos a posturas demarcacionistas en tanto se debería establecer desde esta perspectiva la distinción como disciplina o ciencia.

6. Sin embargo, se debe tener en cuenta que en la producción de conocimiento de cada investigador se tendría que poder observar una epistemología coherente y sólida con los tres componentes del EEPE. Con esto nos referimos a que la perspectiva puede ser, por ejemplo la del pluralismo epistemológico, en la que confluyen varias miradas, pero debe ser clara su posición.

7. Empleamos la categoría de "cabeza vacía" como aquella posición de un investigador que se considera a sí mismo como tabula rasa al momento de comenzar su proceso de investigación.

8. La categoría de "cabeza abierta" es aquella que designa que el investigador pose una visión sobre la realidad, pero tiene apertura en la búsqueda de resultados, está dispuesto a que la realidad no sea como él esperaba que fuese en sus resultados de investigación. 


\section{Referencias}

BACHELARD, G. La formación del espiritu cientifico. México: Siglo XXI editores, 1994.

BALL, S. Sociología das políticas educacionais e pesquisa crítico-social: uma revisão pessoal das políticas educacionais e da pesquisa em política educacional. Currículo sem Fronteiras, v. 6, n. 2, p. 10-32, jul./dez. 2006.

BARRIENTOS DEL MONTE, F. La ciencia política en América Latina: apuntes para una historia (estructural e intelectual) de la disciplina en la región. Instituto Italiano di Scienze Umane, Florencia, Italia. In: SEMINARIO DE INVESTIGACIÓN DEL ÁREA DE CIENCIA POLÍTICA Y DE LA ADMINISTRACIÓN DE LA UNIVERSIDAD DE SALAMANCA, 2009. Anales [...]. Salamanca: Universidad de Salamanca, 2009. http://doi.org/10.13140/RG.2.1.1510.0000

BOBBIO, N. Estado, Gobierno y Sociedad. México: Fondo de Cultura Económica, 1989.

BORÓN, A. Prólogo. In: SOTOLONGO CODINA, P.; DELGADO DÍAZ, C. La revolución contemporánea del saber y la complejidad social. Hacia unas ciencias sociales de nuevo tipo. Buenos Aires: CLACSO, 2006.

BOURDIEU, P. Los usos sociales de la ciencia. Buenos Aires: Nueva Visión, 2000.

BOURDIEU, P. Los usos sociales de la ciencia. Buenos Aires: Nueva Visión, 2003.

BOURDIEU, P.; CHAMBOREDON, J.-C.; PASSERON, J.-C. El oficio de sociólogo. Buenos Aires: Siglo XXI, 2008.

BRAUDEL, F. Histoire et Sciences Sociales: La longue durée. Réseaux, v. 5, n. 27, p. 7-37, 1987. https://doi.org/10.3406/reso.1987.1320

CALDERÓN, F.; PROVOSTE, P. Autonomía, estabilidad y renovación. Los desafíos de las ciencias sociales en América Latina. Buenos Aires: CLACSO, 1992.

CANSINO, C. Adiós a la ciencia política - Crónica de una muerte anunciada. Metapolítica, v. 49, 2006. Disponible en: <http://www.insumisos.com/lecturasinsumisas/ Adios\%20a\%20la\%20ciencia\%20politica.pdf>. Acceso en el: 3 feb. 2009.

CANSINO, C. La muerte de la ciencia política. Buenos Aires: Sudamericana-La Nación, 2008.

CASASSUS, J. Problemas de la gestión educativa en América Latina (la tensión entre los paradigmas de tipo A y el tipo B). Santiago de Chile: Unesco, 2000.

CASTRO-GÓMEZ, S. La reestructuración de las ciencias sociales en América Latina. Bogotá: Instituto PENSAR, 2000. p. 49-70. (Colección Pensar).

COLOMER, J. La ciencia política va hacia adelante (por meandros tortuosos): un comentario a Giovanni Sartori. Política y Gobierno, México, v. 11, n. 2, p. 355-359, 2004.

COVARRUBIAS, I. La ciencia política frente al espejo. Metapolítica, México, n. 14, 2007. Disponible en <https://core.ac.uk/download/pdf/61697664.pdf >. Acceso en el: 8 jul. 2012. 
COX, C. Construcción política de reformas curriculares: el caso de chile en los noventa. Profesorado. Revista de Currículum y Formación del Profesorado, España, v. 10, n. 1, p. 1-24, 2006.

DE SIERRA, G.; GARRETÓN, M. A.; MURMIS, M. TRINDADE, H. Las ciencias sociales en América Latina en una mirada comparativa. In: TRINDADE, H. (org.). Las ciencias sociales en América Latina en perspectiva comparada. México: Siglo XXI, 2006.

GEWIRTZ, S. A reflexividade ética na análise de políticas: conceituação e importância. Práxis Educativa, Ponta Grossa, v. 2, n. 1, p. 7-12, jan./jun. 2007.

GIANELlA, A. Las disciplinas científicas y sus relaciones. Anales de la Educación Común, Buenos Aires, v. 2, n. 3, p. 74-83, abr. 2006.

GLASER, B.; STRAUSS, A. The discovery of grounded theory: strategies for qualitative research. Nueva York: Aldine, 1967.

GÓMEZ CAMPO, V.; TENTI FANFANI, E. Universidad y profesiones. Crisis y alternativas. Buenos Aires: Miño y Dávila, 1989.

GOROSTIAGA, J.; TELLO, C.; ISOLA, N. Investigación educativa en América Latina: Notas históricas y tendencias recientes. In: PALAMIDESSI, M.; SUASNÁBAR, C.; GOROSTIAGA, J. (orgs.) Producción de conocimiento y políticas educativas en América Latina. Argentina: NOVEDUC, 2012.

HABERMAS, J. Ciencia y técnica como ideología. Madrid: Tecnos, 1984.

HABERMAS, J. Conocimiento e interés. Madrid: Taurus, 1981.

KUHN, T. Las estructuras de las revoluciones científicas. Buenos Aires: FCE, 1991.

LAITIN, D. ¿Adónde va la ciencia política? Reflexiones sobre la afirmación del profesor Sartori de que "la ciencia política estadounidense no va a ninguna parte". Política y Gobierno, México, v. 11, n. 2, p. 361-367, 2004.

LANDER, E. “¿Conocimiento para qué? ¿Conocimiento para quién?”. In: CASTRO GÓMEZ, S. La reestructuración de las ciencias sociales en América Latina. Bogotá: Instituto PENSAR, 2000. p. 49-70.

LEBLOND, J. M. La ideología de/en la física contemporánea y otros ensayos críticos. Traducción de Joaquín Jordá. Barcelona: Anagrama, 1975.

LUCCA, J. B. Debates y embates de la politología. Iconos. Revista de Ciencia Sociales, Quito, n. 30, p. 13-26, 2008.

MARTINET, A. C. Epistémologies et sciences de gestion. Paris: Econômica, 1990.

MIÑANA BLASCO, C.; RODRÍGUEZ, J. La educación en el contexto neoliberal. In: BOTERO, D. I. R. (org.). La falacia neoliberal. Crítica y alternativas. Bogotá: Universidad Nacional de Colombia, 2003. p. 285-321.

MOLINA, E. Por una ciencia política enriquecida. Metapolítica, México, n. 49, sept.oct. 2006. Disponible en: <www.insumisos.com/lecturasinsumisas/Por\%20una\%20 ciencia\%20politica\%20enriquecida.pdf $>$. Acceso en el: 10 oct. 2010. 
NAZIF, M.; ROJAS FIGUEROA, A. La investigación educativa latinoamericana en los últimos diez años. Revista de Educación, Madrid, n. 312, p. 21-41, 1997.

NEGRETTO, G. Nota del editor. El rumbo de la ciencia política. Política y Gobierno, México, v. 11, n. 2, p. 347-348, 2004.

ORGANIZACIÓN DE LAS NACIONES UNIDAS PARA LA EDUCACIÓN, LA CIENCIA Y LA CULTURA (UNESCO). In: INTERNATIONAL CONFERENCE ON: METHODS IN POLITICAL SCIENCE, 1948. Anales [...].Paris: UNESCO, 1949.

OXHRON, P. El arte de la ciencia política. Metapolítica, México, n. 49, sept.-oct. 2006. Disponible en: <http://www.insumisos.com/lecturasinsumisas/El\%20arte\%20de $\% 20$ la\%20ciencia\%20\%20politica.pdf>. Acceso en el: 18 dic. 2010.

PAVIGLIANITI, N. Neoconservadurismo y educación: un debate silenciado en la Argentina del 90. Buenos Aires: Libros del Quirquincho, 1993.

PUIGGRÓS, A. Democracia y autoritarismo en la Pedagogía Argentina y Latinoamericana. Buenos Aires: Galerna, 1986.

RAMA, G. Educación, participación y estilos de desarrollo en América Latina. Buenos Aires: Kapelusz - CEPAL, 1984.

SANDÍN, M. P. La enseñanza de la investigación cualitativa. Revista de Enseñanza Universitaria, n. 21, p. 37-52, 2003.

SARTORI, G. ¿Hacia dónde va la ciencia política? Política y Gobierno, México, v. 11, n. 2, p. 349-354, 2004.

SIRE, J. The universe next door: a basic worldview catalogue. Downers Grove, IL: InterVarsity Press, 2004.

SOUSA SANTOS, B. La reinvención del conocimiento y la emancipación social. Buenos Aires: Siglo XXI, CLACSO, 2009.

SOUSA SANTOS, B. La Sociología de las ausencias y la sociología de las emergencias: para una ecología de saberes. In: SOUSA SANTOS, B. Renovar la teoría crítica y reinventar la emancipación social (encuentros en Buenos Aires). Buenos Aires: CLACSO, 2006. p. 13-41.

STREMEL, S. Aspectos teórico-metodológicos para a análise da constituição do campo acadêmico da política educacional no Brasil, RETEPE. Revista de Estudios Teórico y epistemológicos de Política Educativa, v. 2, p. 1-14, 2017. http://dx.doi.org/10.5212/ retepe.v.2.001

SUÁSNABAR, C. Pedagogos críticos, expertos en educación, tecno-políticos, ¿o qué? A propósito de la relación entre intelectuales de la educación y política en los últimos 50 años. In: SEMINARIO ELITES INTELECTUALES Y FORMACIÓN DEL ESTADO, 2009, Buenos Aires. Anales [...]. Buenos Aires, 2009.

TELLO, C. El objeto de estudio en ciencias sociales: entre la pregunta y la hipótesis. Cinta de Moebio, n. 42, p. 225-242, 2011. Disponible en: <https://scielo.conicyt.cl/ scielo.php?script=sci_arttext \&pid=S0717-554X2011000300001\&lng=pt\&nrm=iso $>$. Acceso en el: 12 oct. 2012. 
TELLO, C. Las epistemologías de la política educativa. In: CONGRESO INTERNACIONAL EDUCACIÓN, LENGUAJE Y SOCIEDAD: LA EDUCACIÓN EN LOS NUEVOS ESCENARIOS SOCIOCULTURALES, 2., 2009, La Pampa. Actas... La Pampa, Argentina, 2009.

TELLO, C. Las epistemologías de la política educativa como enfoque y la vigilancia y el posicionamiento epistemológico del investigador. Práxis Educativa, Ponta Grossa, v. 7, n. 1, p. 53-68, jan./jul. 2012. http://dx.doi.org/10.5212/PraxEduc.v.7i1.0003

TELLO, C. Política educativa y fraternidad en América Latina. Notas epistemológicas. In: BARRENECHE, O. (org.). Estudios sobre fraternidad. Buenos Aires: Ciudad Nueva, 2010. p. 187-201. (Serie Política y Sociedad).

TELLO, C.; MAINARDES, J. La posición epistemológica de los investigadores en política educativa: debates teóricos en torno a las perspectivas neo-marxista, pluralista y posestructuralista. Archivos Analíticos de Políticas Educativas, v. 20, n. 9, p. 1-37, 2012. Disponible en: <https://epaa.asu.edu/ojs/article/viewFile/988/942>. Acceso en el: 24 mar. 2013.

TORRES, C. Después de la tormenta neoliberal: la política educativa latinoamericana entre la crítica y la utopía. Revista Iberoamericana de Educación, v. 48, n. 1, p. 207-229, 2008.

VITAR, A. Políticas de educación: razones de una pasión. Buenos Aires: Miño y Dávila, 2006.

WALLERSTEIN, I. Impensar las ciencias sociales. México: Siglo XXI, 1999.

WALLERSTEIN, I. Las incertidumbres del saber. Barcelona: Gedisa, 2004.

WEBER, M. El Político y el científico. Madrid: Alianza Editorial, 1991.

ZOLO, D. La tragedia de la ciencia política. Metapolítica, México, v. 10, n. 49, p. 49-61, sept-oct. 2006. Disponible en: <http://www.temasydebates.unr.edu.ar/index.php/tyd/ article/download/103/102>. Acceso en el: 18 dic. 2011.

\section{SOBRE EL AUTOR}

CÉsar Tello es doctor en Ciencias de la Educación por la Universidad Nacional de La Plata (UNLP). Director de Investigación de la Universidad Nacional de Tres de Febrero (UNTREF).

Recibido en el 28 de junio de 2018.

Aceptado en el 29 de octubre de 2018. 